\title{
Natural Gait Generation Techniques for Principally Kinematic Mechanical Systems
}

\author{
Elie Shammas Howie Choset Alfred Rizzi \\ Carnegie Mellon University, Pittsburgh, PA 15213, U.S.A. \\ eshammas@andrew.cmu.edu_choset@cs.cmu.edu arizzi@cs.cmu.edu
}

\begin{abstract}
In this paper we present a novel gait analysis technique which can directly be used to synthesize gaits for a broad class of mechanical systems. We build upon prior work in locomotion mechanics, however we take a different approach to generate gaits that yield absolute motion of the mechanical system. We present a systematic analysis to control all parameters of a proposed type of gait which eliminates the need for intuition and guesswork as was required in the prior work. The main contribution of the paper is relating position change or motion in the ber space to a volume integral bounded by closed curves on a two dimensional manifold embedded in the base space or shape space of the robot. Not only does our method remove the restriction of using sinusoidal gaits as was the case in the prior work but it also allows for generating optimal gaits by solving a variational problem rather than solving a dynamic programming problem as was the case in the prior work.
\end{abstract}

\section{INTRODUCTION}

The gait generation problem for robotic systems is a dif cult one. Even though researchers have studied this problem extensively, most prior approaches were either empirically derived or considered a restrictive set of possible gaits. Both approaches, however, required some parameter guessing to generate a desired motion. In this paper we simplify the gait generation problem and eliminate not only the restriction of possible gaits but also any guessing of motion parameters for the generated gaits.

To be more speci c, we deal with under-actuated systems, that is, systems that do not have as many actuators as the dimension of their con guration space. Moreover, we are interested in robots that do not have direct control of their position but only of their shape, i.e. we assume that just the shape variables are fully actuated. We address the question of how a multi-bodied robotic system can induce position change upon itself by changing its shape, that is, we address the gait generation problem. Locomotion via shape changes can be found in nature as well: biological snakes locomote by undulating their bodies, and many bacteria locomote by changing their shapes.

In this paper we will generate gaits which produce a desired motion for principally kinematic mechanical systems, that is, systems whose motion is governed solely by the existence of the "right number" of non-holonomic constraints (see Fig. 1). One particular robotic system that we are interested in is the kinematic snake robot (Fig. 1(b)). Not only does this robot have the principally kinematic property but it has been extensively studied in prior literature which allows us to

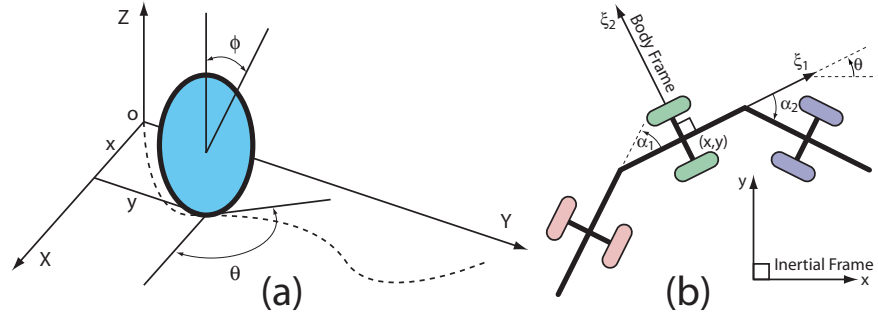

Fig. 1. Con guration variables of the rolling disk (a) and the kinematic snake robot (b).

compare our results to previous work. The work presented in this paper is an extension to our gait generation techniques for purely mechanical systems in [15].

\section{PRIOR WORK}

Con guration space of mechanical systems is usually composed to two spaces, a ber space that represents the position of the robot and a base space that represents the shape of the robot. Gait generation or designing curves in the base space that will produce a desired position change has been extensively studied. Work presented in this paper relates to and builds upon some of this prior research.

\section{A. Bio-mimetic approach:}

During the seventies, Hirose performed extensive experiments on biological snakes to study how they locomote. He was able to de ne a geometric curve, the serpenoid curve, which best approximates the shape of a real snake during undulation [6]. Moreover, Hirose designed and built several robotic snakes which were composed of numerous rigid links that were connected by revolute joints as seen in Fig. 1(a). By attaching passive wheels on the bottom of the links and forcing the robot shape to move along the serpenoid curve, Hirose was able to demonstrate robotic snake locomotion similar to biological snakes locomotion. The restrictions of this approach were that it was system speci $\mathrm{c}$, that is, not generalizable and the parameters of the serponoid curve were empirically ne tuned to only produce smooth snake-like undulation motion.

\section{B. Reduction approach:}

Ostrowski, on the other hand, took advantage of translational symmetry in physics which allowed him to project the entire dynamics of the system onto the shape space, a subspace of the entire con guration space which represents the internal 
shape of the robot. Moreover, by devising a reconstruction equation to relate shape and position velocities he was able to represent the motion of the robot by a non-linear control system. Then by taking recourse to control theory he related the degree of Lie brackets of the control vector elds of the control system to the frequencies of the sinusoidal inputs of the shape variables [13].

Using this approach he was able to intuitively develop and then analyze gaits for several types of mechanical systems including principally kinematic systems. In fact, Ostrowski was able to create gaits for the robot seen in Fig. 1(b) where he had full control of the orientation of the wheel axes as well as the joints' angles. The restriction of this approach was that the inputs had to be sinusoidal; and even though this method produced the frequencies of the sinusoidal inputs, the magnitudes were ne-tuned to produce the desired motion. This method of gait generation is also found in [1], [2], [6] [8], [10],[13], [14], and [16]. In this paper, we consider a simpler kinematic snake by xing the wheel axes' orientations, that is, effectively reducing the shape space dimension. Nonetheless, we are still able to generate gaits that produce the same motions attained in the prior work.

\section{Integration approach:}

We classify mechanical systems into three types: purely mechanical systems, that is, systems whose motions is governed solely by the conservation of momentum laws; principally kinematic systems, that is, systems whose motion is governed solely, by the existence of the right number of independent non-holonomic constraints; and mixed kinematic and dynamic systems, that is, systems whose motion is governed by some non-holonomic constraints and momentum being conserved along some allowable directions. In [15] we generated gaits for purely mechanical systems. The main idea was to relate position change of the robot to a volume integral under a well de ned function.

In this paper we study principally kinematic mechanical systems and we generate gaits for the robotic systems seen in Fig. 1. Mukherjee generated gaits for the rolling disk in a similar approach to the approach that we are presenting in this paper [9]; however, he does not address optimal gaits, nor does he generate gaits from more complex systems. Finally, it is worth mentioning that there has been prior work that directly relates to our approach: [11], [12], and [17]. However, most were concerned with purely mechanical systems and generated gaits only for space robots.

Our gait generation approach is simple and does not have any restrictions on the type of allowable inputs. Hence, for a family of parameterizable gaits, we naturally control all the parameters, frequencies and magnitude, and eliminate the need for deep intuition for nding and ne-tuning these parameters. Moreover, our approach applies to a broad type of mechanical systems.

\section{BACKGROUND MATERIAL}

In this section we introduce principally kinematic systems and several other technical terms that are needed for our gait generation technique.

\section{A. Principal ber bundles:}

We remind the reader that a con guration uniquely speci es the location in two or three dimensions of each physical point of the mechanism or robot. A con guration is expressed by various sets of coordinates that represent the degrees of freedom of the mechanical system. For a robot that is made up of many rigid bodies, both position variables (ber variables) that describe the robot's position with respect to an inertial frame, and shape variables (base variables) that describe the robot's internal angles are needed to specify the robot's con guration.

A general con guration manifold for mechanical systems ${ }^{1}$ is usually denoted by $Q=(G, M)$, where $G$ is the Lie group specifying the position of the robot and $M$ is the base space specifying the shape of the robot. In this paper we deal with con guration manifolds that have a ber bundle structure.

De nition 3.1 (Fiber bundle): A manifold $Q$ with a base subspace $M$ and a projection map $\pi: Q \rightarrow M$ is a ber bundle if for every $r \in M$ there exists a neighborhood $U \subset M$ and $r \in U$ such that $\pi^{-1}(U)$ is homeomorphic to $Y \times U$, where $Y=\pi^{-1}(r)$, that is, locally, $Q \cong Y \times M . Y$ is a ber ${ }^{2}$ which is de ned as the pre-image of $r \in M$ under the map $\pi$.

\section{B. Gait:}

In this paper, we de ne a gait as closed curve, $\gamma(t)$, in the base space, $M$, of the robot. A gait is a mapping

$$
\gamma: \mathbb{R} \rightarrow M,
$$

such that $\gamma(t)=\gamma(t+p) \in M$ where $p \in \mathbb{R}$. Note that for our gait generation technique we require $\gamma(t)$ to be continuous.

\section{Non-holonomic constraints:}

Non-holonomic constraints are constraints that act on conguration velocities and are, by de nition, not integrable. Such constraints are seen in mechanical systems with wheels or rolling elements. The assumption that wheels can not slide sideways nor slip while rolling give rise to non-holonomic constraints. In this paper we will assume that a set of $k$ nonholonomic constraints can be written in a Pfaf an form

$$
\omega(q) \cdot \dot{q}=0,
$$

where $\omega(q)$ is a $k \times n$ matrix describing the constraints and $\dot{q}$ represents an element in the tangent space of the $n$ dimensional con guration manifold $Q$.

\footnotetext{
${ }^{1}$ The con guration space of all mechanical systems have trivial principal bundle structure.

${ }^{2}$ If the ber $Y$ has a group structure then $Q$ is a principal ber bundle, and if $Q=Y \times M$ globally, then $Q$ is a trivial ber bundle.
} 


\section{Kinematic mechanical systems:}

According to [3], a mechanical system is kinematic when its state vector is identical to its con guration vector and the system controls are velocities. For kinematic systems, there is a direct relation between the system's state rst order derivatives and the system's inputs. To be more technical, purely kinematic systems are de ned as systems that have as many independent non-holonomic constraints as the dimension of the system's ber space. The motion of such systems with a con guration $q=\left(g^{l}, r^{m}\right) \in Q^{n=l+m}$ can be described by the reconstruction equation, $\xi=A(g, r) \cdot \dot{r}$, where $\xi$ is the body representation of a $l$-dimensional ber velocity, $A(g, r)$ is an $l \times m$ matrix that depends on the con guration $q$, and $\dot{r}$ is an $m$-dimensional input vector. Moreover, if the non-holonomic constraints of the purely kinematic system are invariant with respect to group action, the mechanical system becomes principally kinematic. Later in the paper we will show how the motion of principally kinematic systems is governed solely by base motions and the prescribed nonholonomic constraints.

De nition 3.2 (Principally kinematic system): Given a mechanical system that has a con guration space with trivial principal ber structure, $Q=G \times M$, where $l$ and $m$ denote the dimensions of $G$ and $M$, respectively, then $n=l+m$ is the dimension of $Q$. Let the mechanical system be subjected to $k$ non-holonomic constraints, $\omega(q) \cdot \dot{q}=0$. Then the dimension of the matrix $\omega$ is $k \times n$. A system is said to be principally kinematic ${ }^{3}$ if $k=l$ (right number of constraints), $\operatorname{det}(\omega(q)) \neq 0$ (linear independence of the constraints), and $\omega(q) \cdot \dot{q}=\omega\left(\Phi_{g}(q)\right) \cdot T_{g} \Phi_{g}(\dot{q})=0$ (invariance of the constraints).

\section{E. Kinematic connections:}

For principally kinematic systems, we can nd a direct relation between shape space velocities, $\dot{r}$, and ber space velocities, $\dot{g}$. This relation is presented in the following Lemma ${ }^{4}$ :

\section{Lemma 3.3 (Local form of kinematic connections):}

Given a principally kinematic mechanical system whose con guration space is a trivial principal bundle $Q^{n}=G^{l} \times M^{m}$, that is, $q=(g, r) \in Q$ and $\dot{q}=(\dot{g}, \dot{r}) \in T_{q} Q$. Moreover, let the system be subject to $k$ linearly independent non-holonomic constraints $\omega(q) \cdot \dot{q}=0$ that are invariant with respect to left group actions ${ }^{5}$. Then we have

$$
T_{g} L_{g^{-1}} \dot{g}=\mathbb{A}(r) \dot{r},
$$

where $T_{g} L_{g^{-1}} \dot{g}$ is the body representation of a ber velocity $\dot{g}, \mathbb{A}(r)$ is a $l \times m$ matrix that is referred to as the local form of the kinematic connection, and $\dot{r}$ is a shape space velocity.

\footnotetext{
${ }^{3}$ Sometimes, these systems are referred to as Chaplygin.

${ }^{4}$ This Lemma can be found in [1] and [13], however, we present a more compact and direct proof in this paper.

${ }^{5}$ For detailed de nitions of some of the terms in this lemma, the reader is referred to [1].
}

Proof: Given the triviality of the con guration space and the invariance of the constraints with respect to left group actions, then we have

$$
\begin{aligned}
\omega(q) \cdot \dot{q} & =\omega\left(\Phi_{h} q\right) \cdot T_{q} \Phi_{h} \dot{q}, \text { or } \\
\omega(g, r) \cdot(\dot{g}, \dot{r})^{T} & =\omega\left(L_{h} g, r\right) \cdot\left(T_{g} L_{h} \dot{g}, \dot{r}\right)^{T},
\end{aligned}
$$

where $\Phi_{h} q$ and $T_{q} \Phi_{h} \dot{q}$ are the left and lifted left actions on the manifold $Q$, [1], [13]. Note that these actions act only on the ber part of $q$. Let $h=g^{-1}$ and using (2) we have

$$
\begin{aligned}
0=\omega(g, r) \cdot(\dot{g}, \dot{r})^{T} & =\omega\left(L_{g^{-1}} g, r\right) \cdot\left(T_{g} L_{g^{-1}} \dot{g}, \dot{r}\right)^{T} \\
& =\omega\left(g^{-1} g, r\right) \cdot\left(T_{g} L_{g^{-1}} \dot{g}, \dot{r}\right)^{T} \\
& =\bar{\omega}(r) \cdot\left(T_{g} L_{g^{-1}} \dot{g}, \dot{r}\right)^{T} .
\end{aligned}
$$

Let $\xi=T_{g} L_{g^{-1}} \dot{g}$ and $\bar{\omega}(r)=\left(\bar{\omega}_{1}(r), \bar{\omega}_{2}(r)\right)$ then writing the above equation in matrix form we get

$$
\left(\begin{array}{ll}
\bar{\omega}_{1}(r) & \bar{\omega}_{2}(r)
\end{array}\right) \cdot\left(\begin{array}{c}
\xi \\
\dot{r}
\end{array}\right)=0 .
$$

Solving for $\xi$ we get

$$
\xi=T_{g} L_{g^{-1}} \dot{g}=\overbrace{\left(\bar{\omega}_{1}(r)\right)^{-1} \cdot \bar{\omega}_{2}(r)}^{-\mathbb{A}(r)} \cdot \dot{r} .
$$

The fact that the non-holonomic constraints are linearly independent implies that $\bar{\omega}_{1}(r)$ is invertible. Also note that $\mathbb{A}(r)$ depends on the base variables only. Hence, by using only the non-holonomic constraints we de ne the kinematic connection seen in (3) which relates the ber velocities to base variables and base velocities. This relation is crucial for our gait generation technique.

\section{F. Exterior algebra:}

We shall review Stokes' theorem in its most general form.

Theorem 3.4 (Stokes' Theorem): Given a one-form $\omega$ and its exterior derivative $d \omega$, we have

$$
\oint_{\partial N} \omega=\int_{N} d \omega,
$$

where $\partial N$ is the boundary of the manifold $N$.

The exterior derivative of a one-form, $\omega=$ $\sum_{i=1}^{m} f_{i}\left(\sigma_{1}, \sigma_{2}, \cdots, \sigma_{m}\right) d \sigma_{i}$, yields a two-form and is given by $d \omega=\sum_{i, j=1, i<j}^{m}\left(\frac{\partial f_{j}}{\partial \sigma_{i}}-\frac{\partial f_{i}}{\partial \sigma_{j}}\right)\left(d \sigma_{i} \wedge d \sigma_{j}\right)$, where $\wedge$ represents the wedge product [4], [7].

\section{Gait Generation}

Now we utilize the de nitions from the previous section to introduce our gait evaluation technique. In (3) we were able to relate base space velocities, $\dot{r}$, to body representation of ber space velocities, $\xi=T_{g} L_{g^{-1}} \dot{g}$ [13], [10]. Each row of (3) can be written as follows:

$$
\xi^{i}=-\sum_{j=1}^{m} \mathbb{A}_{i j}(r) \dot{r}^{j},
$$


where $\mathbb{A}_{i j}(r)$ is the component at the $i$ th row and $j$ th column of the matrix $\mathbb{A}(r)$ and $\dot{r}^{j}$ is the $j$ th component of $\dot{r}$. To compute the change in position, we integrate (5). Note, however, that we are integrating a body representation of a ber velocity. Hence the integral of the left hand side of (5) with respect to time will yield a change in position. This position change is not necessarily an inertial position change as we shall see later in the paper. We de ne a body representation con gur ation variable $\zeta^{i}(t)=\int_{0}^{t} \xi^{i} d t$. As for the right hand term, it is a one-form, hence, we will take its line integral over a path in the base space, then integrating (5) we get

$$
\begin{aligned}
& \Delta \zeta^{i}=\zeta^{i}\left(t_{1}\right)-\zeta^{i}\left(t_{0}\right)=\int_{t_{0}}^{t_{1}} \xi^{i} d t \\
& =\int_{t_{0}}^{t_{1}} \sum_{j=1}^{m}-\mathbb{A}_{i j}(r) \frac{\partial r^{j}}{\partial t} d t \\
& =\quad \oint_{\gamma\left(t_{0}\right)}^{\gamma\left(t_{1}\right)} \sum_{j=1}^{m}-\mathbb{A}_{i j}(r) d r^{j}, \\
& =\quad \iint_{\Gamma_{k, j=1, k<j}} \sum_{\left(\frac{\partial \mathbb{A}_{i j}(r)}{\partial r_{k}}-\frac{\partial \mathbb{A}_{i k}(r)}{\partial r_{j}}\right)}^{F^{i}}\left(d r_{k} \wedge d r_{j}\right), \\
& =\quad \sum_{k, j=1, k<j}^{m} \iint_{\Gamma} F^{i}\left(r_{1}, r_{2}, \cdots, r_{m}\right)\left(d r_{k} \wedge d r_{j}\right), \quad \text { (6) }
\end{aligned}
$$

where $\gamma(t)$ is a closed one-dimensional curve in the base space and $\Gamma$ is the interior of that curve on an arbitrary twodimensional smooth manifold embedded in the base space. Note that the integrand functions $F^{i}$ de ned in the last row of the previous equation are functions of the base variables only. The above equation relates position change in body representations, $\Delta \zeta^{i}$, to a sum of volume integrals.

By studying the integrands in the above equation and by designing and placing curves in the base space we are able to generate gaits that will move the mechanical system in a desired direction. Finally, we remind the reader that $\Delta \zeta$ is an integral of a body representation of a ber velocity $\xi$. Hence, it does not necessarily relate trivially to inertial position change. This is the case only when the ber has a trivial group structure, that is the lifted action map is the identity map, $T_{g} L_{g^{-1}} \dot{g}=\dot{g}$.

\section{A. Properties of height functions}

By studying certain properties of the integrand functions $F^{i}$ we are able to evaluate closed curves in the base space. We shall refer to the integrand functions as height functions ${ }^{6}$ in the rest of the paper.We shall study the following properties of the height functions.

1) Symmetry:: We study periodicity which allows us to investigate smaller portions of the base space. Moreover, we nd the set of points about which the height function is odd.

\footnotetext{
${ }^{6}$ Note that we have $l$ height functions where $l$ is the dimension of the ber space $G$.
}

A gait that is symmetric about an odd point of the height function yields zero ber motion while a gait that changes orientation as it passes through an odd point is guaranteed to have a non-zero position change.

2) Signed regions:: Since we are integrating a height function over a closed region, it is important to know where the height function is positive, negative, or zero. Not only does this allows us to control the direction of motion along the ber but also to optimize gaits by restricting them to lie in a strictly positive or negative region.

3) Unboundedness:: While designing gaits, one should stay away from regions where the height function tends to in nity. A gait that passes through such regions may yield in nite volume, that is, in nite position change for nite shape changes. Usually this is an indication that a non-holonomic constraint is being violated.

By inspecting the above properties of the height functions we are able to evaluate the position change of the robot due to following any closed curve in the base space.

\section{B. Gait generation with height functions}

We have related motion along the ber to an oriented volume integral in the base space. Hence, we can compute how the robot's position changes as it changes its shape along any closed curve in the base space. Since our gait evaluation technique is direct and simple we can devise a set of simple rules that can be used to generate gaits.

1) Closed non-self-intersecting curves:: Any closed nonself-intersecting curve that lies entirely in a positive or negative region is guaranteed to produce a non-zero ber motion.

2) Closed self-intersecting curves:: Any closed selfintersecting curve is guaranteed to produce a non-zero ber motion provided that the curve spans more than one region, the self-intersection occurs on a boundary edge between two regions of opposite signs, and the orientation of the curve changes sign as it crosses from one region to another.

3) Symmetric curves around points in the set K:: Assuming that the height function is hyper-odd over the set of points in $K$, then any curve symmetric with respect to points in $K$ will enclose equal areas in two adjacent regions that have opposite signs. Integrating the volume under such curves will yield zero, that is, the ber motion for such gaits is identically zero.

These rules do not add any constraints on the shape of the input curves. For instance, as long as the curve stays entirely in one region and does not intersect itself, it is guaranteed to generate a nonzero ber motion. The bigger the area enclosed by the curve within a positive or negative region, the bigger the generated phase shift. This relaxes the restriction of sinusoidal inputs that was required in prior work.

\section{Optimal gaits}

Earlier we related position change of the robot to an oriented volume under a well-de ned height function. By changing the shape and position of a gait, $\gamma(t)$, we are able to change the magnitude of the ber motion. In this section we formulate a 
variational problem that maximizes the ber motion magnitude for all gaits with a given length. We refer the reader to [5] for a detailed description of the calculus of variations ideas used here.

For optimality analysis it is easier to work with line integrals seen in (3) rather than dealing with double integrations. Hence, the functional which we are optimizing is a one-form

$$
J(\gamma)=\oint_{\gamma(t)} \omega .
$$

To simplify the mathematical notation, let $\omega$ be two dimensional, that is,

$$
\omega=f\left(r_{1}, r_{2}\right) d r_{1}+g\left(r_{1}, r_{2}\right) d r_{2}
$$

where $r_{1}, r_{2}$ are the base variables.

Note that the requirement that the gaits have a $\mathrm{x}$ ed length is essential. If such a bound is not enforced we can get unbounded volume for a gait with unbounded length. For this problem it is convenient to parameterize the gait by its arc length, $s$. This will yield a well de ned functional that we are trying to maximize

$$
\max J(\gamma(s))=\int_{s_{0}}^{s_{1}}\left(f\left(r_{1}, r_{2}\right) \frac{\partial r_{1}}{\partial s}+g\left(r_{1}, r_{2}\right) \frac{\partial r_{2}}{\partial s}\right) d s .
$$

In this problem we have an additional constraint on the length of the curves; this can be represented by the following integral:

$$
L=\int_{s_{0}}^{s_{1}} \sqrt{\left(\partial r_{1} / \partial s\right)^{2}+\left(\partial r_{2} / \partial s\right)^{2}} d s .
$$

The above two integrals de ne a constrained calculus of variations problem. We resort to numerical methods to optimize our gaits. However, the main bene $t$ from our gait analysis technique is that now we can solve for optimal gaits using a variational problem. This is a signi cant accomplishment compared to the prior work where a dynamic programming problem was used.

\section{EXAMPLES}

In this section we will generate gaits for two principally kinematic systems, the rolling disk and the kinematic snake.

\section{A. Rolling disk:}

The rolling disk has a con guration manifold $Q=\mathbb{R} \times \mathbb{R} \times$ $\mathbb{S} \times \mathbb{S}$. Let $q=(x, y, \theta, \phi) \in Q$ denote the disk con guration where $(x, y)$ represent the location of the point of contact, $\theta$ represents the orientation angle measured from the $x$-axis, and $\phi$ represents the rolling angle measured from the vertical axis (Fig. 1(a)). We will assume that we have full actuation control of both the steering and rolling angles. Hence, the base space is $M=\mathbb{S} \times \mathbb{S}$ and the ber space is $G=\mathbb{R} \times \mathbb{R}$ which is two-dimensional. Additionally we have two non-holonomic constraints: no sideways sliding $(\dot{x} \sin \theta-\dot{y} \cos \theta=0)$ and rolling without slipping $(\dot{x} \cos \theta+\dot{y} \sin \theta-R \dot{\phi}=0)$. Solving for the ber velocities $\dot{x}$ and $\dot{y}$ using the constraints and writing the solution in matrix form we get
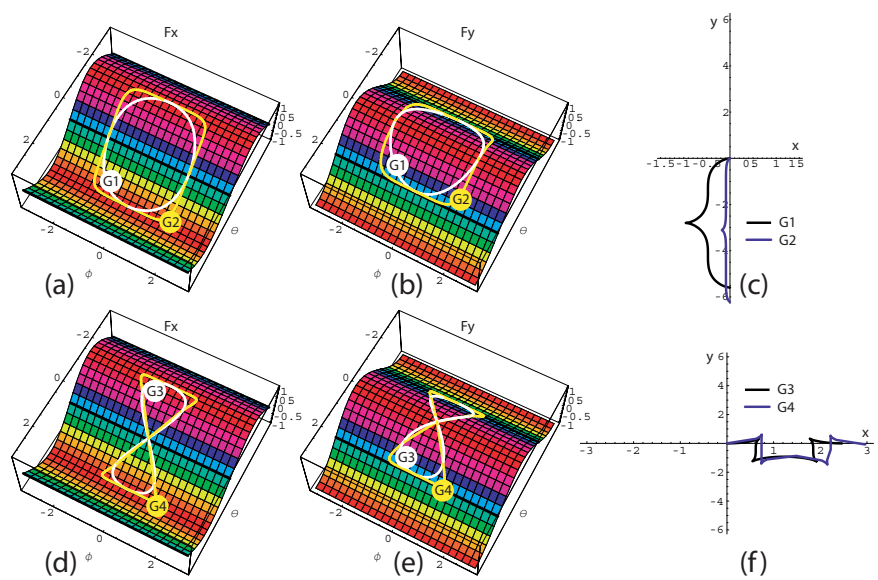

(f)

Fig. 2. The rolling disk height functions along the $x$ direction are seen in (a)\&(d) and the $y$ direction in (b)\&(e). Four types of gaits are simulated and their respective trace for the contact point is seen in (c)\&(f).

$$
\underbrace{\left(\begin{array}{c}
\xi^{1} \\
\xi^{2}
\end{array}\right)=\left(\begin{array}{c}
\dot{x} \\
\dot{y}
\end{array}\right)}_{T_{g} L_{g^{-1}} \dot{g} \text { for }}=\underbrace{\left(\begin{array}{ll}
0 & R \cos \theta \\
0 & R \sin \theta
\end{array}\right)}_{\mathbb{A} \times \mathbb{R}(r)}\left(\begin{array}{c}
\dot{\theta} \\
\dot{\phi}
\end{array}\right) .
$$

Using Equation 6, we can compute the change in both the $x$ and $y$ directions for any gait $\gamma(t)=(\theta(t), \phi(t))$

$$
\begin{aligned}
\Delta x & =\Delta \zeta^{1}=\oint_{\gamma} R \cos (\theta) d \phi \\
& =\iint_{\Gamma} \underbrace{-R \sin (\theta)}_{F_{x}(\theta, \phi)} d \theta d \phi, \\
\Delta y & =\Delta \zeta^{2}=\oint_{\gamma} R \sin (\theta) d \phi \\
& =\iint_{\Gamma} \underbrace{R \cos (\theta)}_{F_{y}(\theta, \phi)} d \theta d \phi .
\end{aligned}
$$

A three-dimensional plot of both height functions can be seen in Fig. 2(a) and 2(b). Let us study the properties of these speci c height functions for the rolling disk. Symmetry: Both $F_{x}$ and $F_{y}$ are periodic with period $2 \pi$, hence it suf ces to look at the region $(\theta, \phi) \in[-\pi, \pi] \times[-\pi, \pi]$. Moreover, the height functions are quite simple, both are independent of the rolling variables, $\phi$. Plus, the height functions inherit the odd and even properties from the sine and cosine functions in the orientation variable, $\theta$. Zero curves: We can easily compute the zero lines, $(\theta, \phi)=(t, k \pi), k \in \mathbb{Z}$ for $F_{x}$ and $(\theta, \phi)=(t,(2 k+1) \pi / 2), k \in \mathbb{Z}$ for $F_{y}$. These lines identify the positive and negative regions for both height functions. Boundedness: Note that there are no in nity lines for the rolling disk example. The reason is that the non-holonomic constraints can never be linearly dependent.

Given the above properties of the height functions for the rolling disk, gait generation is direct. Moreover, since the ber 
group is $\mathbb{R} \times \mathbb{R}$ and is Abelian ${ }^{7}$, we can relate the position change in both the $x$ and $y$ directions directly to the volume integrals as seen in Equations 7 and 8. (This trivial relation is not true in general, as we shall see in the second example in this paper.)

To generate a gait that moves the disk in the $y$ direction only, a circular gait that stays in a positive region of $F_{y}$ while being symmetric to a zero line of $F_{x}$ will produce this motion, that is, $\Delta x=0, \Delta y>0$ as seen in Fig. 2(a,b). We could shift this gait along the $\theta$ direction to produce motion in the $x$ direction, or we can use a "gure- 8 " type of gait to produce the same net motion, that is, $\Delta x>0, \Delta y=0$ as seen in Fig. 2(d,e). These two families of gaits allow us to move the rolling disk in any direction in the plane. Also note that we are not bound to use sinusoidal gaits. We simulated two other types of gait as seen in Fig. 2(c,f). In fact, we achieved bigger magnitudes for these gaits when compared to the sinusoidal counterparts.

\section{B. Kinematic snake robot:}

Now we will generate gaits for a more complex mechanical system, the kinematic snake (Fig. 1(b)). Ostrowski was able to generate gaits for a similar system [13], however, he had full control over the orientation of the wheel axes as well as the joint angles, that is, making the base space ve-dimensional. In this paper, we have actuation of the joints only and $\mathrm{x}$ the wheel axes to the links without compromising the overall mobility of the robot, that is, even though the base space is two-dimensional, we are still able to generate gaits that will move the robot along the body representation of all ber directions.

The con guration space of the kinematic snake has a principal bundle structure and is ve-dimensional, $Q=G \times M=$ $S E(2) \times(\mathbb{S} \times \mathbb{S})$. Let $q=\left(x, y, \theta, \alpha_{1}, \alpha_{2}\right) \in Q$ represent the robot's con guration and $2 L$ be the length of each link. Since we have three sets of passive wheels, three non-holonomic constraints with no sideways slipping are generated, that is,

$$
\begin{aligned}
-S_{\theta+\alpha_{1}} \dot{x}+C_{\theta+\alpha_{1}} \dot{y}-L \dot{\alpha}_{1}-L\left(1+C_{\alpha_{1}}\right) \dot{\theta} & =0, \\
-S_{\theta} \dot{x}+C_{\theta} \dot{y} & =0, \\
-S_{\theta-\alpha_{2}} \dot{x}+C_{\theta-\alpha_{2}} \dot{y}-L \dot{\alpha}_{2}+L\left(1+C_{\alpha_{2}}\right) \dot{\theta} & =0,
\end{aligned}
$$

where $C_{*}=\cos (*)$ and $S_{*}=\sin (*)$. Solving the above system for $\dot{g}=\{\dot{x}, \dot{y}, \dot{\theta}\}$ and then solving for the body representation $\xi=T_{g} L_{g^{-1}} \dot{g}$ we get

\footnotetext{
${ }^{7}$ For Abelian groups, $T_{g} L_{g^{-1}}$ is the identity map, that is, $\xi=T_{g} L_{g^{-1}} \dot{g}=$
}

$$
\begin{aligned}
& \xi= \overbrace{\left(\begin{array}{ccc}
\cos \theta & \sin \theta & 0 \\
-\sin \theta & \cos \theta & 0 \\
0 & 0 & 1
\end{array}\right)}^{T_{g} L_{g-1} \text { for } G=S E(2)} \overbrace{\left(\begin{array}{c}
\dot{x} \\
\dot{y} \\
\dot{\theta}
\end{array}\right)}^{\dot{g}}= \\
& \underbrace{\left(\begin{array}{cc}
\frac{L\left(1+C_{\alpha_{2}}\right) \dot{\alpha}_{1}}{S_{\alpha_{1}}+S_{\alpha_{1}-\alpha_{2}}-S_{\alpha_{2}}} & \frac{L\left(1+C_{\alpha_{1}}\right) \dot{\alpha}_{2}}{\left(1+S_{\alpha_{2}}\right) S_{\alpha_{1}}-\left(1+C_{\alpha_{1}}\right) S_{\alpha_{2}}} \\
0 & 0 \\
\frac{-S_{\alpha_{2} \dot{\alpha}_{1}}}{S_{\alpha_{1}}+S_{\alpha_{1}-\alpha_{2}}-S_{\alpha_{2}}} & \frac{-S_{\alpha_{1}} \dot{\alpha}_{2}}{\left(1+S_{\alpha_{2}}\right) S_{\alpha_{1}}-\left(1+C_{\alpha_{1}}\right) S_{\alpha_{2}}}
\end{array}\right)}_{\mathbb{A}(r)} \dot{r} .
\end{aligned}
$$

Note that as expected $\mathbb{A}(r)$ is a function of the base space variables. This is due to the constraints being invariant with respect to group actions when represented in body coordinates. Then we take each row of the above equation and integrate it over a period of a cyclic gait. By using Stokes' Theorem we convert the line integrals to volume integrals which compute the ber motion in the directions $\left\{\xi_{1}, \xi_{2}, \xi_{3}\right\}$.

$$
\begin{aligned}
\Delta \zeta^{1} & =\iint_{\Gamma} \underbrace{\left(\frac{L}{-1+\cos \left(\alpha_{1}-\alpha_{2}\right)}\right)}_{F_{1}\left(\alpha_{1}, \alpha_{2}\right)} d \alpha_{1} d \alpha_{2}, \\
\Delta \zeta^{2} & =\iint_{\Gamma} \underbrace{0}_{F_{2}\left(\alpha_{1}, \alpha_{2}\right)} d \alpha_{1} d \alpha_{2} \equiv 0, \\
\Delta \zeta^{3} & =\iint_{\Gamma} \underbrace{\left(\frac{\tan \left(\frac{\alpha_{1}}{2}\right)+\tan \left(\frac{\alpha_{2}}{2}\right)}{4 \sin ^{2}\left(\frac{\alpha_{1}-\alpha_{2}}{2}\right)}\right)}_{F_{3}\left(\alpha_{1}, \alpha_{2}\right)} d \alpha_{1} d \alpha_{2} .
\end{aligned}
$$

The ber motion in the $\xi^{2}$ direction from (10) is identically zero for any gait. This agrees with the physical intuition that the middle link can not move in the $\xi^{2}$ direction without breaking the "no sideways motion" constraint of the wheels in the middle link (Fig. 1(a)). But we can still generate gaits that will produce motion in the other two directions $\xi^{1}$ and $\xi^{3}$ by studying the properties of their associated height functions $F_{1}$ and $F_{3}$ given in (9) and (11).

Inspecting the height functions in (9) and (11), we deduce the following properties: Symmetry: Since $\alpha_{1}, \alpha_{2} \in \mathbb{S}$, both $F_{1}$ and $F_{3}$ are periodic with period $2 \pi$, hence it suf ces to look at the region $\left(\alpha_{1}, \alpha_{2}\right) \in[-\pi, \pi] \times[-\pi, \pi]$. Moreover, $F_{1}$ has two types of symmetry, $F_{1}\left(\alpha_{1}, \alpha_{2}\right)=F_{1}\left(\alpha_{1}+\right.$ $\left.c, \alpha_{2}+c\right)$ for $c \in \mathbb{R}$ and $F_{1}$ is even about its in nity lines. As for $F_{3}$ is odd about its zero lines. Zero curves: Note that $F_{1}$ is never zero while the zero lines for $F_{3}$ are $\left(\alpha_{1}, \alpha_{2}\right)=(t,-t+2 k \pi), k \in \mathbb{Z}$. Boundedness: We can easily compute the in nity lines, $\left(\alpha_{1}, \alpha_{2}\right)=(t, t+2 k \pi), k \in \mathbb{Z}$ for $F_{1}$ and $\left(\alpha_{1}, \alpha_{2}\right)=(t, t+2 k \pi),(t, k \pi),(k \pi, t), k \in \mathbb{Z}$ for $F_{3}$. The zero and in nity lines identify the positive and negative regions for both height functions. To be able to plot these unbounded height functions, we used the arctan function to map the unbounded values to $\pm \pi / 2$. The plots of the integrands are seen in Fig. 3(a)\&(b). 
The unbounded lines described above represent singular con gurations of the robot. So if the snake robot starts from a con guration on these lines, the robot will not be able to move away from it without breaking a non-holonomic constraint. Moreover, if a region enclosed within a designed gait contains a portion of the above lines, the computed ber motion for such a gait might be unbounded. Physically, this means that for a nite shape motion, the robot will have in nite motion which can be understood as breaking a constraint. Hence, not only do the height functions help us design gaits but also identify regions where constraints are singular. Having identi ed some useful properties of the integrand functions, we can move on to designing gaits.

Given the above properties, we can design gaits that guarantee a non-zero ber motion in either the $\xi^{1}, \xi^{3}$, or both the $\xi^{1}$ and $\xi^{3}$ directions.

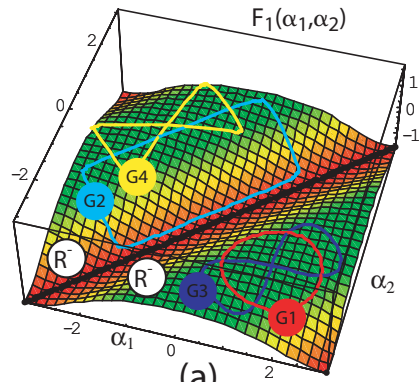

(a)
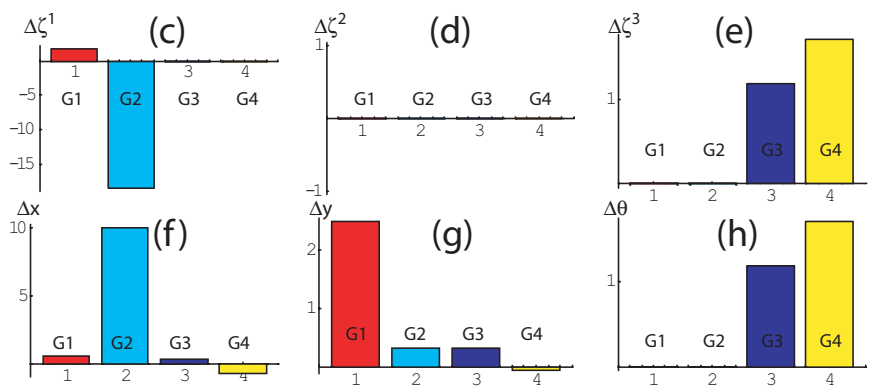

Fig. 3. Four designed gaits in (a) and (b) with the respective ber motion magnitudes in (c)-(h). $G 1$ and $G 3$ are sinusoidal while $G 2$ and $G 4$ are not. For example, for $G 2$ we have $\{\Delta x \neq 0, \Delta y \neq 0, \Delta \theta=0\}$ while $\left\{\Delta \zeta^{1} \neq\right.$ $\left.0, \Delta \zeta^{2}=0, \Delta \zeta^{3}=0\right\}$.

Two non-self-intersecting gaits, $G 1$ and $G 3$, can be seen in Fig. 3(a)\&(b). As expected these gaits have zero phase shift in the $\xi^{2}$ and $\xi^{3}$ directions but a non-zero phase shift in the $\xi^{1}$ direction (Fig. 3(c)-(e)). Similarly, Fig. 3(a)\&(b) depict selfintersecting gaits. These gaits generate zero ber motion in the $\xi^{1}$ and $\xi^{2}$ directions but a non-zero ber motion in the $\xi^{3}$ direction (Fig. 3(c)-(e)).

Note that the generated ber motion is computed in body representation; however, we would like to compute the phase shift in the generalized coordinate representation. As seen in Fig. 3(c)-(h), $\Delta \zeta^{1} \neq \Delta x$ and $\Delta \zeta^{2} \neq \Delta y$ while $\Delta \zeta^{3}=\Delta \theta$. This discrepancy in the computed ber motion values is due to the body representation, which depends on the group structure of the ber space. For this example, the group structure is
$S E(2)$, and the relation is as follows:

$$
\begin{aligned}
& \Delta \zeta^{1}=\int_{t_{0}}^{t_{1}} \xi^{1} d t=\int_{t_{0}}^{t_{1}} \dot{x} \cos \theta+\dot{y} \sin \theta d t, \\
& \Delta \zeta^{2}=\int_{t_{0}}^{t_{1}} \xi^{2} d t=\int_{t_{0}}^{t_{1}}-\dot{x} \sin \theta+\dot{y} \cos \theta d t, \\
& \Delta \zeta^{3}=\int_{t_{0}}^{t_{1}} \xi^{3} d t=\int_{t_{0}}^{t_{1}} \dot{\theta} d t=\Delta \theta .
\end{aligned}
$$
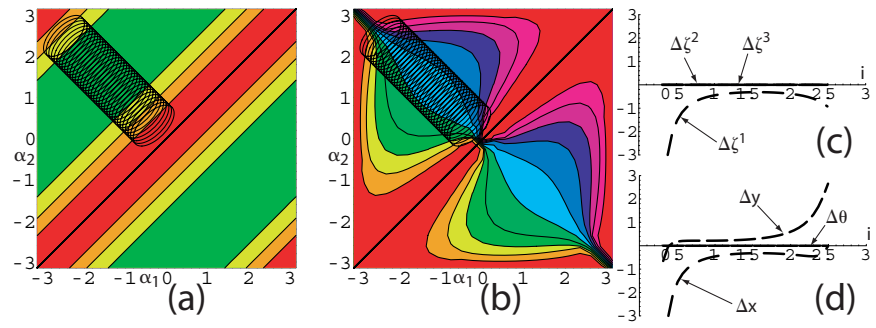

Fig. 4. Variations in the ber motion as an elliptical gait is moved over height functions $F_{1}$ and $F_{3}$ in (a) and (b) respectively. We measure the motion $\{\Delta x, \Delta y, \Delta \theta\}$ and $\left\{\Delta \zeta^{1}, \Delta \zeta^{2}, \Delta \zeta^{3}\right\}$ in (c) and (d) respectively. $i$ in (c) and (d) represents the distance between the center of the ellipses and the origin.

To understand the difference between the two representations and to get a hint on how to resolve this difference we present the following example. Consider an elliptical gait whose major axis is aligned with the line $\alpha_{2}=\alpha_{1}$ and whose minor axis is aligned with the line $\alpha_{2}=-\alpha_{1}$. The center of the elliptical gait is moved along the line $\alpha_{2}=-\alpha_{1}$ as seen in Fig. 4(a) and (b). As we move the gait, we numerically compute the ber motion along the directions, $(x, y, \theta)$. Then we use integration to compute the ber motion in the body representations along the $\left(\xi^{1}, \xi^{2}, \xi^{3}\right)$ directions. As expected from our analysis of our gait generation techniques, all gaits have a non-zero ber motion only in the $\xi^{1}$ direction (Fig. $4(c)$ ). However, the inertial position change is different as seen in Fig. 4(d). Note that only the $\theta$ direction which is identically zero matches the $\xi^{3}$ direction. This is due to the group structure of $S E(2)$ where $\Delta \zeta^{3}=\Delta \theta$ as seen in (14).

Another important thing to notice is that as the gaits approach the origin $\left(\alpha_{1}, \alpha_{2}\right)=(0,0)$, the motion in the $x$ direction dominates while as the gaits approach the point $\left(\alpha_{1}, \alpha_{2}\right)=(\pi, \pi)$, the motion in the $y$ direction dominates (Fig. 4(d)). So we can control our gaits to produce motion solely along each of the ber variables $\{x, y, \theta\}$.

\section{DEMONSTRATIONS}

We built a simple three-link kinematic snake robot out of Legos ${ }^{\circledR}$ and two hobby servos to actuate the two joints. We implemented our gaits on this experimental snake robot and achieved good results. In Fig. 5(a) we implemented a gait that will produce a net motion in the $x$ direction. The robot's locomotion was in the the $x$ direction but shifted a bit in the $y$ direction as well. We relate this to the some minor observed slipping in the wheels and other mechanical imperfections of 


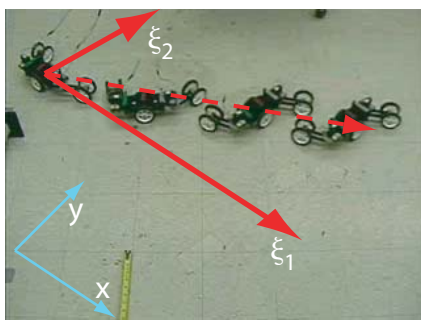

(a)

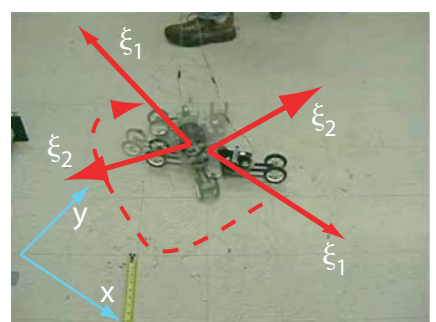

(b)
Fig. 5. Translation and rotation experiments for the kinematic snake.

the robot. Similarly we implemented a rotation gait as seen in Fig. 5(b). The snake robot rotated in place as expected, however the origin of the body frame slightly deviated. Again we claim that this was caused by the reasons stated above.

\section{CONCLUSION}

We presented a solution to the problem of gait generation for principally kinematic mechanical systems. Prior work in this area realized the same result, but with the limitation of sinusoidal inputs. Moreover, the prior work required some guesswork and intuition in specifying the input parameters. The main contribution of this paper is to use Stokes' theorem to relate integrals along closed paths in the base space to motions in the ber space. This allowed for a systematic analysis and control over the input parameters, eliminating the need for any guesswork in specifying these parameters.

Moreover, our gait analysis tool identi es the presence of singularities in the constraints. Prior work has identi ed such singularities; however, our method, by studying the right type of properties such as symmetry, periodicity, boundedness, and sign region, not only identi es these singularities but also produces a richer set of allowable gaits. Finally, it is worth mentioning that our method allows us to solve for optimal gaits by solving a variational problem.

Thus far we have been able to generate gait for purely mechanical systems [15] and principally kinematic systems as presented in this paper. We have been working on the third type of system, mixed dynamic and non-holonomic system. We believe that our approach should tackle this third type of systems as we have already had some promising results.

\section{ACKNOWLEDGMENT}

We would like to thank Joel Burdick, David Conner, Vijay Kumar, Kevin Lynch, and Jim Ostrowski for their feedback and input into this work. Finally, we want to thank the anonymous reviewers who have provided valuable inputs and feedback that helped improve this paper.

\section{REFERENCES}

[1] A. Bloch. Nonholonomic Mechanics and Control. Springer Verlag, 2003.

[2] F. Bullo and A. D. Lewis. Geometric Control of Mechanical Systems: Modeling, Analysis, and Design for Simple Mechanical Control Systems. Springer, 2004.

[3] H. Choset, K.M. Lynch, S. Hutchinson, G. Kantor, W. Burgard, L.E. Kavraki, and S. Thrun. Principles of Robot Motion: Theory, Algorithms, and Implementations. The MIT Press, 2005.

[4] R. W.R. Darling. Differential Forms and Connections. Cambridge University Press, 1994.

[5] M. D. Foss and W. J. Hrusa. Lecture Notes: Calculus of Variations. 2004.

[6] S. Hirose. Biologically Inspired Robots (Snake-like Locomotor and Manipulator). Oxford University Press, 1993.

[7] G. Lugo. Differntial geometry in physics. Technical report, University of North Carolina, 1998.

[8] J. Marsden. Introduction to Mechanics and Symmetry. Springer-Verlag, 1994.

[9] R. Mukherjee and D.P. Anderson. Nonholonomic motion planning using stoke's theorem. In IEEE International Conference on Robotics and Automation, 1993.

[10] R. M. Murray and S. S. Sastry. Nonholonomic motion planning: Steering using sinusoids. IEEE T. Automatic Control, 38(5):700 - 716, May 1993.

[11] Y. Nakamura and R. Mukherjee. Nonholonomic path planning of space robots. In IEEE International Conference on Robotics and Automation, 1989.

[12] Y. Nakamura and R. Mukherjee. Nonholonomic path planning of space robots via a bidirectional approach. In IEEE Transactions on Robotics and Autmation, volume 7, pages 500-514, 1991.

[13] J. Ostrowski and J. Burdick. The mechanics and control of undulatory locomotion. International Journal of Robotics Research, 17(7):683 701, July 1998.

[14] J. Ostrowski, J. Desai, and V. Kumar. Optimal gait selection for nonholonomic locomotion systems. International Journal of Robotics Research, 2000.

[15] E. Shammas, K. Schmidt, and H. Choset. Natural gait generation techniques for multi-bodied isolated mechanical systems. In IEEE International Conference on Robotics and Automation, 2005.

[16] G. Walsh and S. Sastry. On reorienting linked rigid bodies using internal motions. Robotics and Automation, IEEE Transactions on, 11(1):139146, January 1995.

[17] K. Yamada. Arm path planning for a space robot. In IEEE/RSJ International Conference on Intelligent Robots and Systems, 1993. 